\title{
Two hypotheses on the exponential class in the class of $O$-subexponential infinitely divisible distributions
}

\author{
Toshiro Watanabe \\ Center for Mathematical Sciences, The University of Aizu, Aizu-Wakamatsu, \\ Fukushima 965-8580, Japan \\ E-mail: t-watanb@u-aizu.ac.jp
}

\begin{abstract}
Two hypotheses on the class $\mathcal{L}(\gamma)$ in the class $\mathcal{O S} \cap \mathcal{I D}$ are discussed. Two weak hypotheses on the class $\mathcal{L}(\gamma)$ in the class $\mathcal{O S} \cap \mathcal{I D}$ are proved. A necessary and sufficient condition in order that, for every $t>0$, the $t$-th convolution power of a distribution in the class $\mathcal{O S} \cap \mathcal{I D}$ belongs to the class $\mathcal{L}(\gamma)$ is given. Sufficient conditions are given for the validity of two hypotheses on the class $\mathcal{L}(\gamma)$.
\end{abstract}

Key words : exponential class, $O$-subexponentiality, infinite divisibility, convolution roots.

Mathematics Subject Classification : 60E07 60G51

\section{Introduction and results}

In what follows, we denote by $\mathbb{R}$ the real line and by $\mathbb{R}_{+}$the half line $[0, \infty)$. Denote by $\mathbb{N}$ the totality of positive integers and by $a \mathbb{N}$ the set $\{a, 2 a, 3 a, \ldots\}$. The symbol $\delta_{a}(d x)$ stands for the delta measure at $a \in \mathbb{R}$. Let $\eta$ and $\rho$ be probability distributions on $\mathbb{R}$. We denote by $\eta * \rho$ the convolution of $\eta$ and $\rho$ and by $\rho^{n *} n$-th convolution power of $\rho$ with the understanding that $\rho^{0 *}(d x)=$ $\delta_{0}(d x)$. Denote by $\bar{\xi}(x)$ the tail $\xi((x, \infty))$ of a measure $\xi$ on $\mathbb{R}$ for $x \in \mathbb{R}$. Let $\gamma \geq 0$. We define the $\gamma$-exponential moment $\widehat{\xi}(\gamma)$ as

$$
\widehat{\xi}(\gamma):=\int_{-\infty}^{\infty} e^{\gamma x} \xi(d x) .
$$

If $\widehat{\xi}(\gamma)<\infty$, we define the Fourier-Laplace transform $\widehat{\xi}(\gamma+i z)$ for $z \in \mathbb{R}$ as

$$
\widehat{\xi}(\gamma+i z):=\int_{-\infty}^{\infty} e^{(\gamma+i z) x} \xi(d x)
$$

An integral $\int_{a}^{b} g(x) \rho(d x)$ means $\int_{a+}^{b+} g(x) \rho(d x)$. For positive functions $f_{1}(x)$ and $g_{1}(x)$ on $[A, \infty)$ for some $A \in \mathbb{R}$, we define the relation $f_{1}(x) \sim g_{1}(x)$ by $\lim _{x \rightarrow \infty} f_{1}(x) / g_{1}(x)=1$ and the relation $f_{1}(x) \asymp g_{1}(x)$ by

$$
0<\liminf _{x \rightarrow \infty} f_{1}(x) / g_{1}(x) \leq \limsup _{x \rightarrow \infty} f_{1}(x) / g_{1}(x)<\infty .
$$

Let $\gamma \geq 0$. A distribution $\rho$ on $\mathbb{R}$ belongs to the class $\mathcal{L}(\gamma)$ if $\bar{\rho}(x)>0$ for all $x>0$ and, for every $a \in \mathbb{R}$,

$$
\bar{\rho}(x+a) \sim e^{-\gamma a} \bar{\rho}(x) .
$$


A distribution $\rho$ on $\mathbb{R}$ belongs to the class $\mathcal{S}(\gamma)$ if $\rho \in \mathcal{L}(\gamma), \widehat{\rho}(\gamma)<\infty$, and

$$
\overline{\rho^{2 *}}(x) \sim 2 \widehat{\rho}(\gamma) \bar{\rho}(x) .
$$

A distribution $\rho$ on $\mathbb{R}$ belongs to the class $\mathcal{O L}$ if $\bar{\rho}(x)>0$ for $x>0$ and, for all $a \geq 0$,

$$
\bar{\rho}(x-a) \asymp \bar{\rho}(x) .
$$

A distribution $\rho$ on $\mathbb{R}$ belongs to the class $\mathcal{O S}$ if $\bar{\rho}(x)>0$ for all $x>0$ and

$$
\overline{\rho^{2 *}}(x) \asymp \bar{\rho}(x) .
$$

Note that the class $\mathcal{O S}$ is included in the class $\mathcal{O} \mathcal{L}$. A distribution $\rho$ on $\mathbb{R}$ belongs to the class $\mathcal{S}_{\sharp}$ if $\rho \in \mathcal{O S}$ and

$$
\limsup _{A \rightarrow \infty} \limsup _{x \rightarrow \infty} \frac{\bar{\rho}(x-A) \bar{\rho}(A)+\int_{A}^{x-A} \bar{\rho}(x-u) \rho(d u)}{\bar{\rho}(x)}=0 .
$$

The class $\mathcal{S}_{\sharp}$ includes $\cup_{\gamma \geq 0} \mathcal{S}(\gamma)$ and it is closed under convolution powers. A finite measure $\xi$ satisfies the Wiener condition if $\widehat{\xi}(i z) \neq 0$ for every $z \in \mathbb{R}$. Denote by $\mathcal{W}$ the totality of finite measures on $\mathbb{R}$ satisfying the Wiener condition. We denote by $\mathcal{I D}$ the class of all infinitely divisible distributions on $\mathbb{R}$. For $\mu \in \mathcal{I D}$, denote by $\nu$ its Lévy measure. Under the assumption that $\bar{\nu}(c)>0$ for every $c>0$, define $\nu_{1}(d x):=1_{(1, \infty)}(x) \nu(d x) / \bar{\nu}(1)$. Let $\mu \in \mathcal{I D}$. We define a compound Poisson distribution $\mu_{1}$ with $c=\bar{\nu}(1)$ as

$$
\mu_{1}(d x):=e^{-c} \sum_{k=0}^{\infty} \frac{c^{k}}{k !} \nu_{1}^{k *}(d x) .
$$

Denote by $\mu^{t *}$ the $t$-th convolution power of $\mu \in \mathcal{I D}$ for $t>0$. Note that $\mu^{t *}$ is the distribution of $X_{t}$ for a certain Lévy process $\left\{X_{t}\right\}$ on $\mathbb{R}$. Let $\gamma \geq 0$. Define $T(\mu, \gamma)$ as

$$
T(\mu, \gamma):=\left\{t>0: \mu^{t *} \in \mathcal{L}(\gamma)\right\}
$$

Since the class $\mathcal{L}(\gamma)$ is closed under convolutions by Theorem 3 of Embrechts and Goldie [2, $T(\mu, \gamma)$ is empty or an additive semigroup in $(0, \infty)$. We see from Lemma 2.2 below that for $\mu \in \mathcal{O S} \cap \mathcal{I D}$, there are positive integers $n$ such that $\nu_{1}^{n *} \in \mathcal{O S}$. Let $n_{0}$ be the positive integer defined by (2.1) below. Note that we do not yet know an example of $\mu \in \mathcal{O S} \cap \mathcal{I D}$ such that $n_{0} \geq 3$.

A class $\mathcal{C}$ of distributions is called closed under convolution roots if $\rho^{n *} \in \mathcal{C}$ for some $n \in \mathbb{N}$ implies $\rho \in \mathcal{C}$. We see from Shimura and Watanabe 11] that the class $\mathcal{O S}$ is not closed under convolution roots, but from Watanabe and Yamamuro [15] that the class $\mathcal{O S} \cap \mathcal{I D}$ is closed under convolution roots. Embrechts et al. 4] in the one-sided case and Watanabe [13] in the two-sided case proved that the class $\mathcal{S}(0)$ is closed under convolution roots and Embrechts and Goldie 2] conjectured that the class $\mathcal{L}(\gamma)$ with $\gamma \geq 0$ is closed under convolution roots, but Shimura and Watanabe [12] showed that the class $\mathcal{L}(\gamma)$ with $\gamma \geq 0$ is not closed under convolution roots. Moreover, Watanabe and Yamamuro [16] proved that the class $\mathcal{S}_{a c}$ of all absolutely continuous distributions on $\mathbb{R}$ with subexponential densities is not closed under convolution roots. Embrechts and Goldie [3] conjectured that the class $\mathcal{S}(\gamma)$ with $\gamma>0$ is closed under convolution roots. Watanabe 13 proved that $\mathcal{S}(\gamma) \cap \mathcal{I D}$ with $\gamma \geq 0$ is closed 
under convolution roots, but Watanabe [14 showed that the class $\mathcal{S}(\gamma)$ with $\gamma>0$ is not closed under convolution roots. We add the following. Klüppelberg [5] showed that the class $\mathcal{O S}$ is closed under convolutions. The class $\mathcal{S}(\gamma)$ is closed under convolution powers for $\gamma \geq 0$, but Leslie [7, for $\gamma=0$, and Klüppelberg and Villasenor [6], for $\gamma>0$, proved that the class $\mathcal{S}(\gamma)$ is not closed under convolutions.

We consider the following two hypotheses on the class $\mathcal{L}(\gamma)$ in the class $\mathcal{O S} \cap \mathcal{I D}:$

Hypothesis I. Let $\gamma \geq 0$. For every $\mu \in \mathcal{O S} \cap \mathcal{I D}$, if $\mu^{n *} \in \mathcal{L}(\gamma)$ for some $n \in \mathbb{N}$, then $\mu^{(n+1) *} \in \mathcal{L}(\gamma)$.

HYротнESIS II. Let $\gamma \geq 0$. For every $\mu \in \mathcal{O S} \cap \mathcal{I D}$, if $\mu^{n *} \in \mathcal{L}(\gamma)$ for some $n \in \mathbb{N}$, then $\mu \in \mathcal{L}(\gamma)$.

We also consider the weak version of the above hypotheses :

Hypothesis I'. Let $\gamma \geq 0$. For every $\mu \in \mathcal{O S} \cap \mathcal{I D}$, if $\mu^{n *}, \mu^{(n+1) *} \in \mathcal{L}(\gamma)$ for some $n \in \mathbb{N}$, then $\mu^{(n+2) *} \in \mathcal{L}(\gamma)$.

Hypothesis II'. Let $\gamma \geq 0$. For every $\mu \in \mathcal{O S} \cap \mathcal{I D}$, if $\mu^{n *}, \mu^{(n+1) *} \in \mathcal{L}(\gamma)$ for some $n \in \mathbb{N}$, then $\mu \in \mathcal{L}(\gamma)$.

Let $\gamma \geq 0$. Define

$$
\begin{gathered}
\mathcal{A}(\gamma):=\{\mu \in \mathcal{O S} \cap \mathcal{I D}: T(\mu, \gamma)=(0, \infty)\} \\
\mathcal{B}(\gamma):=\{\mu \in \mathcal{O S} \cap \mathcal{I D}: T(\mu, \gamma)=\emptyset\}
\end{gathered}
$$

and

$$
\mathcal{C}(\gamma):=\left\{\mu \in \mathcal{O S} \cap \mathcal{I D}: T(\mu, \gamma)=a_{0} \mathbb{N} \text { with some } a_{0}>0\right\} .
$$

Theorem 1.1. Let $\gamma \geq 0$ and $\mu \in \mathcal{O S} \cap \mathcal{I D}$. We have the following:

(i) $\mathcal{O S} \cap \mathcal{I D}=\mathcal{A}(\gamma) \cup \mathcal{B}(\gamma) \cup \mathcal{C}(\gamma)$. Thus Hypotheses I' and II' are true.

(ii) The relation $\mu \in \mathcal{A}(\gamma)$ holds if and only if, for all $a \geq 0$,

$$
\lim _{x \rightarrow \infty} \frac{e^{-\gamma a} \overline{\overline{\nu_{1}}}(x-a)-\overline{\nu_{1}}(x)}{\overline{\nu_{1}^{n_{0} *}}(x)}=0 .
$$

If $\mu \in \mathcal{A}(\gamma)$, then $\nu_{1}^{n *} \notin \mathcal{L}(\gamma) \cap \mathcal{O S}$ for $1 \leq n \leq n_{0}-1$ and $\nu_{1}^{n *} \in \mathcal{L}(\gamma) \cap \mathcal{O S}$ for $n \geq n_{0}$.

Corollary 1.1. Let $\gamma \geq 0$. Then the following are equivalent:

(1) Hypothesis I is true.

(2) Hypothesis II is true.

(3) $\mathcal{C}(\gamma)$ is empty.

(4) For every $\mu \in \mathcal{O S} \cap \mathcal{I D}$ it holds that, for every $2 t \in T(\mu, \gamma)$ and for every $a \geq 0$,

$$
\limsup _{x \rightarrow \infty} \limsup _{\lambda \rightarrow \infty} \frac{\left|\int_{x}^{\lambda-x}\left(e^{-\gamma a} \overline{\mu_{1}^{t *}}(\lambda-a-u)-\overline{\mu_{1}^{t *}}(\lambda-u)\right) \mu_{1}^{t *}(d u)\right|}{\overline{\mu_{1}^{t *}}(\lambda)}=0 .
$$

Remark 1.1. Let $\gamma=0$. Then, $\mathcal{C}(0)$ is empty and Hypotheses I and II are true. The relation $\mu \in \mathcal{A}(0)$ holds if and only if

$$
\lim _{x \rightarrow \infty} \frac{\nu_{1}((x, x+1])}{\overline{\nu_{1}^{n_{0} *}}(x)}=0 .
$$


If $\mu \in \mathcal{A}(0)$, then $\nu_{1}^{n *} \notin \mathcal{L}(0) \cap \mathcal{O S}$ for $1 \leq n \leq n_{0}-1$ and $\nu_{1}^{n *} \in \mathcal{L}(0) \cap \mathcal{O S}$ for $n \geq n_{0}$. Xu et al. showed in Theorem 2.2 of [18] an example of $\mu \in \mathcal{A}(0)$ with $n_{0}=2$.

For $\gamma>0$, we cannot yet answer the question whether Hypotheses I and II are true. However, under some additional assumptions in terms of Lévy measure, we establish that $\mathcal{C}(\gamma)$ is empty.

Proposition 1.1. Let $\gamma>0$ and $\mu \in \mathcal{O S} \cap \mathcal{I D}$. Suppose that, for every $a \geq 0$,

$$
\liminf _{x \rightarrow \infty} e^{-\gamma a} \bar{\nu}_{1}(x-a) / \bar{\nu}_{1}(x) \geq 1 .
$$

Then, we have either $T(\mu, \gamma)=(0, \infty)$ or $\emptyset$.

Remark 1.2. Cui et al. [1] proved a result analogous to the above proposition under a stronger assumption. Xu et al. showed in Theorem 1.1 of [19] an example of the case where $T(\mu, \gamma) \neq \emptyset$ in the above proposition.

Proposition 1.2. Let $\gamma>0$ and $\mu \in \mathcal{O S} \cap \mathcal{I D}$. Suppose that $\nu_{1}^{2 *} \in \mathcal{L}(\gamma)$ and the real part of $\widehat{\nu}_{1}(\gamma+i z)$ is not zero for every $z \in \mathbb{R}$. Then, either $T(\mu, \gamma)=(0, \infty)$ or $\emptyset$.

Proposition 1.3. Let $\gamma>0$ and $\mu \in \mathcal{O S} \cap \mathcal{I D}$. Suppose that there exists $n_{1} \in \mathbb{N}$ such that $\nu_{1}^{n_{1} *} \in \mathcal{S}_{\sharp}$. Then, either $T(\mu, \gamma)=(0, \infty)$ or $\emptyset$. The equality $T(\mu, \gamma)=(0, \infty)$ holds if and only if $\nu_{1} \in \mathcal{S}(\gamma)$.

Remark 1.3. Watanabe made in Theorem 1.1 of 14 a distribution $\eta \in \mathcal{S}_{\sharp}$ such that $\eta^{n *} \in \mathcal{S}(\gamma)$ for every $n \geq 2$ but $\eta \notin \mathcal{S}(\gamma)$. Thus, taking this $\eta$ as $\nu_{1}$, then Proposition 1.3 holds with $T(\mu, \gamma)=\emptyset$.

\section{Preliminaries}

In this section, we give several basic results as preliminaries. Pakes 8 proved the following.

Lemma 2.1. (Lemmas 2.1 and 2.5 of [8]) Let $\mu \in \mathcal{I D}$. Then we have $\mu \in \mathcal{L}(\gamma)$ if and only if $\mu_{1} \in \mathcal{L}(\gamma)$.

Watanabe and Yamamuro [15] proved the following.

Lemma 2.2. (Proposition 3.1 of [15]) Suppose that $\mu \in \mathcal{I D}$. Then, we have $\mu \in \mathcal{O S}$ if and only if there is $n \in \mathbb{N}$ such that $\nu_{1}^{n *} \in \mathcal{O S}$ and $\overline{\mu_{1}^{t *}}(x) \asymp \overline{\nu_{1}^{n *}}(x)$ for any $t>0$.

For $\mu \in \mathcal{O S} \cap \mathcal{I D}$, define $n_{0} \in \mathbb{N}$ as

$$
n_{0}:=\min \left\{n \in \mathbb{N}: \nu_{1}^{n *} \in \mathcal{O S}\right\} .
$$

Lemma 2.3. Let $\mu \in \mathcal{O S} \cap \mathcal{I D}$.

(i) There exists $C(a)>0$ such that, for all $a \geq 0$ and all $x>0$,

$$
\overline{\nu_{1}^{n_{0 *}}}(x-a) \leq C(a) \overline{\nu_{1}^{n_{0 *}}}(x) .
$$

(ii) There exists $K>1$ such that, for all $n \in \mathbb{N}$ and all $x>0$,

$$
\overline{\nu_{1}^{n *}}(x) \leq K^{n} \overline{\nu_{1}^{n_{0} *}}(x) .
$$


Proof. Assertion (i) is clear since $\nu_{1}^{n_{0} *} \in \mathcal{O S} \subset \mathcal{O} \mathcal{L}$. We see from Proposition 2.4 of Shimura and Watanabe [1] that there exists $K_{1}>1$ such that, for all $k \in \mathbb{N}$ and all $x>0$,

$$
\overline{\nu_{1}^{\left(k n_{0}\right) *}}(x) \leq K_{1}^{k} \overline{\nu_{1}^{n_{0} *}}(x) .
$$

Note that, for $m \leq n$,

$$
\overline{\nu_{1}^{m *}}(x) \leq \overline{\nu_{1}^{n *}}(x) .
$$

Hence, we have, for $0 \leq j \leq n_{0}-1$ and for all $k \in \mathbb{N}$, with $K=K_{1}^{2 / n_{0}}>1$

$$
\overline{\nu_{1}^{\left(k n_{0}+j\right) *}}(x) \leq K^{\left(k n_{0}+j\right)} \overline{\nu_{1}^{n_{0} *}}(x) .
$$

This inequality holds for $k=0$ too. Thus assertion (ii) is true.

Under the assumption that $\zeta \in \mathcal{O S} \subset \mathcal{O} \mathcal{L}$, we define the following. Let

$$
d^{*}:=\limsup _{x \rightarrow \infty} \frac{\overline{\zeta^{2 *}}(x)}{\bar{\zeta}(x)}<\infty .
$$

Let $\Lambda$ be the totality of increasing sequences $\left\{\lambda_{n}\right\}_{n=1}^{\infty}$ with $\lim _{n \rightarrow \infty} \lambda_{n}=\infty$ such that, for every $x \in \mathbb{R}$, the following limit exists and is finite:

$$
m\left(x ;\left\{\lambda_{n}\right\}\right):=\lim _{n \rightarrow \infty} \frac{\bar{\zeta}\left(\lambda_{n}-x\right)}{\bar{\zeta}\left(\lambda_{n}\right)} .
$$

Define, for each sequence $\left\{x_{n}\right\}_{n=1}^{\infty}$ with $\lim _{n \rightarrow \infty} x_{n}=\infty, T_{n}(y)$ as

$$
T_{n}(y):=\frac{\bar{\zeta}\left(x_{n}-y\right)}{\bar{\zeta}\left(x_{n}\right)} .
$$

Since $\left\{T_{n}(y)\right\}_{n=1}^{\infty}$ is a sequence of increasing functions, uniformly bounded on every finite interval, by Helly's selection principle, there exists an increasing subsequence $\left\{\lambda_{n}\right\}$ of $\left\{x_{n}\right\}$ with $\lim _{n \rightarrow \infty} \lambda_{n}=\infty$ such that everywhere on $\mathbb{R}$ (2.2) holds. The limit function $m\left(x ;\left\{\lambda_{n}\right\}\right)$ is increasing and is finite. That is, $\left\{\lambda_{n}\right\} \in \Lambda$. It follows that, under the assumption that $\zeta \in \mathcal{O S}$, there exists an increasing subsequence $\left\{\lambda_{n}\right\} \in \Lambda$ of $\left\{x_{n}\right\}$ for each sequence $\left\{x_{n}\right\}_{n=1}^{\infty}$ with $\lim _{n \rightarrow \infty} x_{n}=\infty$.

Lemma 2.4. Suppose that $\zeta \in \mathcal{O S}$. Then, we have the following.

(i) If $\left\{\lambda_{n}\right\} \in \Lambda$, then $\left\{\lambda_{n}-a\right\} \in \Lambda$ for every $a \in \mathbb{R}$.

(ii) For $\left\{\lambda_{n}\right\} \in \Lambda$,

$$
\int_{-\infty}^{\infty} m\left(x ;\left\{\lambda_{n}\right\}\right) \zeta(d x)<\infty
$$

and

$$
\lim _{a \rightarrow \infty} m\left(a ;\left\{\lambda_{n}\right\}\right) \bar{\zeta}(a)=0 .
$$

In particular, if $\zeta \in \mathcal{O S} \cap \mathcal{L}(\gamma)$, then $m\left(x ;\left\{\lambda_{n}\right\}\right)=e^{\gamma x}$ and $\widehat{\zeta}(\gamma)<\infty$.

Proof. We prove (i). Suppose that $\left\{\lambda_{n}\right\} \in \Lambda$. We have, for $x, a \in \mathbb{R}$,

$$
\lim _{n \rightarrow \infty} \frac{\bar{\zeta}\left(\lambda_{n}-a-x\right)}{\bar{\zeta}\left(\lambda_{n}-a\right)}=\frac{m\left(x+a ;\left\{\lambda_{n}\right\}\right)}{m\left(a ;\left\{\lambda_{n}\right\}\right)} .
$$


Thus $\left\{\lambda_{n}-a\right\} \in \Lambda$. Next, we prove (ii). Let $\rho$ be a distribution on $\mathbb{R}$. Note that, for $x>2 A$,

$$
\overline{\rho^{2 *}}(x)=2 \int_{-\infty}^{A+} \bar{\rho}(x-u) \rho(d u)+\bar{\rho}(x-A) \bar{\rho}(A)+\int_{A}^{x-A} \bar{\rho}(x-u) \rho(d u) .
$$

We see from (2.3) that, for $\left\{\lambda_{n}\right\} \in \Lambda$ and $s>0$,

$$
\begin{aligned}
d^{*} & \geq \limsup _{n \rightarrow \infty} \frac{\overline{\zeta^{2 *}}\left(\lambda_{n}\right)}{\bar{\zeta}\left(\lambda_{n}\right)} \\
& \geq 2 \limsup _{n \rightarrow \infty}^{s+} \frac{\bar{\zeta}\left(\lambda_{n}-x\right)}{\bar{\zeta}\left(\lambda_{n}\right)} \zeta(d x) \\
& \geq 2 \int_{-\infty}^{s+} m\left(x ;\left\{\lambda_{n}\right\}\right) \zeta(d x) .
\end{aligned}
$$

As $s \rightarrow \infty$, we have

$$
\int_{-\infty}^{\infty} m\left(x ;\left\{\lambda_{n}\right\}\right) \zeta(d x)<\infty .
$$

Since $m\left(x ;\left\{\lambda_{n}\right\}\right)$ is increasing in $x$, we have

$$
\begin{aligned}
& \lim _{a \rightarrow \infty} m\left(a ;\left\{\lambda_{n}\right\}\right) \bar{\zeta}(a) \\
& \leq \lim _{a \rightarrow \infty} \int_{a+}^{\infty} m\left(x ;\left\{\lambda_{n}\right\}\right) \zeta(d x)=0 .
\end{aligned}
$$

Hence, if $\zeta \in \mathcal{O S} \cap \mathcal{L}(\gamma)$, then $m\left(x ;\left\{\lambda_{n}\right\}\right)=e^{\gamma x}$ and $\widehat{\zeta}(\gamma)<\infty$. Thus we have proved the lemma.

Pakes [8, 9] asserted and Watanabe [13] finally proved the following.

Lemma 2.5. (Theorem 1.1 of [13]) Let $\gamma \geq 0$. Then $\mu \in \mathcal{I D} \cap \mathcal{S}(\gamma)$ if and only if $\nu_{1} \in \mathcal{S}(\gamma)$.

Lemma 2.6. Let $\gamma \geq 0$. Suppose that $\rho \in \mathcal{S}_{\sharp}$.

(i) If $\bar{\eta}(x) \asymp \bar{\rho}(x)$, then $\eta \in \mathcal{S}_{\sharp}$.

(ii) $\rho \in \mathcal{S}(\gamma)$ if and only if $\rho \in \mathcal{L}(\gamma)$.

Proof. Suppose that $\rho \in \mathcal{S}_{\sharp}$. We prove (i). If $\bar{\eta}(x) \asymp \bar{\rho}(x)$, then there is $C>0$ such that $\bar{\eta}(x) \leq C \bar{\rho}(x)$ for $x \in \mathbb{R}$. By using integration by parts in the second inequality, we obtain that

$$
\begin{aligned}
& \bar{\eta}(x-A) \bar{\eta}(A)+\int_{A}^{x-A} \bar{\eta}(x-u) \eta(d u) \\
& \leq C^{2} \bar{\rho}(x-A) \bar{\rho}(A)+C \int_{A}^{x-A} \bar{\rho}(x-u) \eta(d u) \\
& \leq 2 C^{2} \bar{\rho}(x-A) \bar{\rho}(A)+C^{2} \int_{A}^{x-A} \bar{\rho}(x-u) \rho(d u) .
\end{aligned}
$$

Thus, we see that

$$
\limsup _{A \rightarrow \infty} \limsup _{x \rightarrow \infty} \frac{\left(\bar{\eta}(x-A) \bar{\eta}(A)+\int_{A}^{x-A} \bar{\eta}(x-u) \eta(d u)\right)}{\bar{\eta}(x)}=0 .
$$


That is, $\eta \in \mathcal{S}_{\sharp}$. Next we prove (ii). If $\rho \in \mathcal{S}(\gamma)$, then clearly $\rho \in \mathcal{L}(\gamma)$. Note that, for $x>2 A,(2.3)$ holds. If $\rho \in \mathcal{S}_{\sharp} \cap \mathcal{L}(\gamma)$, then we have

$$
\begin{aligned}
& \lim _{x \rightarrow \infty} \frac{\overline{\rho^{2 *}}(x)}{\bar{\rho}(x)} \\
= & \lim _{A \rightarrow \infty} 2 \int_{-\infty}^{A+} \lim _{x \rightarrow \infty} \frac{\bar{\rho}(x-u)}{\bar{\rho}(x)} \rho(d u) \\
= & 2 \widehat{\rho}(\gamma)<\infty .
\end{aligned}
$$

Thus we see that $\rho \in \mathcal{S}(\gamma)$.

Watanabe [14] extended Wiener's approximation theorem in [17] as follows.

Lemma 2.7. (Lemma 2.6 of Watanabe [14]) Let $\xi$ be a finite measure on $\mathbb{R}$. The following are equivalent:

(1) $\xi \in \mathcal{W}$.

(2) If, for a bounded measurable function $g(x)$ on $\mathbb{R}$,

$$
\int_{-\infty}^{\infty} g(x-t) \xi(d t)=0 \text { for a.e. } x \in \mathbb{R}
$$

then $g(x)=0$ for a.e. $x \in \mathbb{R}$.

\section{Convolution lemmas}

In this section, we give important lemmas on convolutions.

Lemma 3.1. Let $\gamma \geq 0$. Suppose that $\zeta \in \mathcal{O S}$. For $j=1,2$, let $\rho_{j}$ be distributions on $\mathbb{R}_{+}$satisfying

$$
\bar{\rho}_{j}(x) \leq C_{j} \bar{\zeta}(x) \text { with some } C_{j}>0 \text { for all } x>0 .
$$

Let $\left\{\lambda_{n}\right\} \in \Lambda$.

(i) Let $\lambda_{n}>a+x$ and $x>0$. We have, for every $a \geq 0$,

$$
e^{-\gamma a} \overline{\rho_{1} * \rho_{2}}\left(\lambda_{n}-a\right)-\overline{\rho_{1} * \rho_{2}}\left(\lambda_{n}\right)=: \sum_{j=1}^{4} I_{j},
$$

where

$$
\begin{gathered}
I_{1}:=-\int_{\lambda_{n}-a-x}^{\lambda_{n}-x} \overline{\overline{\rho_{1}}}\left(\lambda_{n}-y\right) \rho_{2}(d y), \\
I_{2}:=\overline{\rho_{1}}(x)\left(e^{-\gamma a \overline{\rho_{2}}}\left(\lambda_{n}-a-x\right)-\overline{\rho_{2}}\left(\lambda_{n}-x\right)\right), \\
I_{3}:=\int_{0-}^{\left(\lambda_{n}-a-x\right)+}\left(e^{-\gamma a} \overline{\rho_{1}}\left(\lambda_{n}-a-y\right)-\overline{\rho_{1}}\left(\lambda_{n}-y\right)\right) \rho_{2}(d y),
\end{gathered}
$$

and

$$
I_{4}:=\int_{0-}^{x+}\left(e^{-\gamma a} \overline{\rho_{2}}\left(\lambda_{n}-a-y\right)-\overline{\rho_{2}}\left(\lambda_{n}-y\right)\right) \rho_{1}(d y) .
$$

(ii) We have for $j=1,2$

$$
\limsup _{x \rightarrow \infty} \limsup _{n \rightarrow \infty} \frac{\left|I_{j}\right|}{\bar{\zeta}\left(\lambda_{n}\right)}=0 .
$$


Proof. By using integration by parts, we have

$$
\begin{aligned}
& \overline{\rho_{1} * \rho_{2}}\left(\lambda_{n}-a\right) \\
& =\int_{0-}^{\left(\lambda_{n}-a-x\right)+} \overline{\rho_{1}}\left(\lambda_{n}-a-y\right) \rho_{2}(d y) \\
& +\int_{\lambda_{n}-a-x}^{\lambda_{n}-a} \overline{\rho_{1}}\left(\lambda_{n}-a-y\right) \rho_{2}(d y)+\overline{\rho_{2}}\left(\lambda_{n}-a\right) \\
& =\int_{0-}^{\left(\lambda_{n}-a-x\right)+} \overline{\rho_{1}}\left(\lambda_{n}-a-y\right) \rho_{2}(d y)+\int_{0-}^{x+} \overline{\rho_{2}}\left(\lambda_{n}-a-y\right) \rho_{1}(d y) \\
& +\overline{\rho_{1}}(x) \overline{\rho_{2}}\left(\lambda_{n}-a-x\right),
\end{aligned}
$$

and

$$
\begin{aligned}
& \overline{\rho_{1} * \rho_{2}}\left(\lambda_{n}\right) \\
& =\int_{0-}^{\left(\lambda_{n}-a-x\right)+} \overline{\rho_{1}}\left(\lambda_{n}-y\right) \rho_{2}(d y)+\int_{\lambda_{n}-a-x}^{\lambda_{n}-x} \overline{\rho_{1}}\left(\lambda_{n}-y\right) \rho_{2}(d y) \\
& +\int_{\lambda_{n}-x}^{\lambda_{n}} \overline{\rho_{1}}\left(\lambda_{n}-y\right) \rho_{2}(d y)+\overline{\rho_{2}}\left(\lambda_{n}\right) \\
& =\int_{0-}^{\left(\lambda_{n}-a-x\right)+} \overline{\rho_{1}}\left(\lambda_{n}-y\right) \rho_{2}(d y)+\int_{\lambda_{n}-a-x}^{\lambda_{n}-x} \overline{\rho_{1}}\left(\lambda_{n}-y\right) \rho_{2}(d y) \\
& +\int_{0-}^{x+} \overline{\rho_{2}}\left(\lambda_{n}-y\right) \rho_{1}(d y)+\overline{\rho_{1}}(x) \overline{\rho_{2}}\left(\lambda_{n}-x\right) .
\end{aligned}
$$

Thus assertion (i) is valid. We have by Lemma 2.4 for $j=1,2$

$$
\begin{aligned}
& \limsup _{x \rightarrow \infty} \limsup _{n \rightarrow \infty} \frac{\left|I_{j}\right|}{\bar{\zeta}\left(\lambda_{n}\right)} \\
& \leq \limsup _{x \rightarrow \infty} \overline{\rho_{1}}(x) \limsup _{n \rightarrow \infty} \frac{\overline{\rho_{2}}\left(\lambda_{n}-a-x\right)}{\bar{\zeta}\left(\lambda_{n}\right)} \\
& \leq C_{1} C_{2} \limsup _{x \rightarrow \infty} \bar{\zeta}(x) m\left(x ;\left\{\lambda_{n}-a\right\}\right) m\left(a ;\left\{\lambda_{n}\right\}\right)=0 .
\end{aligned}
$$

Lemma 3.2. Let $\gamma \geq 0$. Suppose that $\zeta \in \mathcal{O S}$. For $j=1,2$, let $\rho_{j}$ be distributions on $\mathbb{R}_{+}$satisfying (3.1). Suppose further that for $j=1,2$ and every $a \geq 0$,

$$
\lim _{x \rightarrow \infty} \frac{e^{-\gamma a} \bar{\rho}_{j}(x-a)-\bar{\rho}_{j}(x)}{\bar{\zeta}(x)}=0 .
$$

Then, for every $a \geq 0$,

$$
\lim _{x \rightarrow \infty} \frac{e^{-\gamma a \overline{\rho_{1} * \rho_{2}}}(x-a)-\overline{\rho_{1} * \rho_{2}}(x)}{\bar{\zeta}(x)}=0 .
$$

Proof. Let $\left\{\lambda_{n}\right\} \in \Lambda$. By the assumption for $j=1$, there is $\epsilon(x)>0$ such that $\epsilon(x) \rightarrow 0$ as $x \rightarrow \infty$ and

$$
\left|e^{-\gamma a} \overline{\rho_{1}}\left(\lambda_{n}-a-y\right)-\overline{\rho_{1}}\left(\lambda_{n}-y\right)\right| \leq \epsilon(x) \zeta\left(\lambda_{n}-y\right)
$$


for $0 \leq y \leq \lambda_{n}-a-x$. Thus we have

$$
\begin{aligned}
& \limsup _{x \rightarrow \infty} \limsup _{n \rightarrow \infty} \frac{\left|I_{3}\right|}{\bar{\zeta}\left(\lambda_{n}\right)} \\
& \leq \limsup _{x \rightarrow \infty} \epsilon(x) \limsup _{n \rightarrow \infty} \frac{\int_{0-}^{\left(\lambda_{n}-a-x\right)+\bar{\zeta}\left(\lambda_{n}-y\right) \rho_{2}(d y)}}{\bar{\zeta}\left(\lambda_{n}\right)} \\
& \leq \limsup _{x \rightarrow \infty} \epsilon(x) \limsup _{n \rightarrow \infty} \frac{\bar{\zeta}\left(\lambda_{n}\right)+\int_{a+x}^{\lambda_{n}} \overline{\rho_{2}}\left(\lambda_{n}-y\right) \zeta(d y)}{\bar{\zeta}\left(\lambda_{n}\right)} \\
& \leq \limsup _{x \rightarrow \infty} \epsilon(x) \limsup _{n \rightarrow \infty} \frac{\bar{\zeta}\left(\lambda_{n}\right)+C_{2} \overline{\zeta^{2 *}}\left(\lambda_{n}\right)}{\bar{\zeta}\left(\lambda_{n}\right)}=0 .
\end{aligned}
$$

As in the above argument, we have

$$
\limsup _{x \rightarrow \infty} \limsup _{n \rightarrow \infty} \frac{\left|I_{4}\right|}{\bar{\zeta}\left(\lambda_{n}\right)}=0 .
$$

Thus, by (3.2) and (3.3) of Lemma 3.1, we have proved (3.4).

Lemma 3.3. Let $\gamma \geq 0$. Suppose that $\zeta \in \mathcal{O S}$. For $j=1,2$, let $\rho_{j}$ be distributions on $\mathbb{R}_{+}$satisfying (3.1). Suppose further that, for $j=1,2$, and for every $a \geq 0$,

$$
\liminf _{x \rightarrow \infty} \frac{e^{-\gamma a} \bar{\rho}_{j}(x-a)-\bar{\rho}_{j}(x)}{\bar{\zeta}(x)} \geq 0 .
$$

Then, we have, for every $a \geq 0$,

$$
\liminf _{x \rightarrow \infty} \frac{e^{-\gamma a} \overline{\rho_{1} * \rho_{2}}(x-a)-\overline{\rho_{1} * \rho_{2}}(x)}{\bar{\zeta}(x)} \geq 0 .
$$

Proof. Let $\left\{\lambda_{n}\right\} \in \Lambda$. Let $\epsilon>0$ and $a \geq 0$ be arbitrary and let $n \in \mathbb{N}$ and $x \in\left(0, \lambda_{n}-a\right)$ be sufficiently large such that

$$
e^{-\gamma a} \overline{\rho_{1}}\left(\lambda_{n}-a-y\right)-\overline{\rho_{1}}\left(\lambda_{n}-y\right) \geq-\epsilon \bar{\zeta}\left(\lambda_{n}-y\right)
$$

for $0 \leq y \leq \lambda_{n}-a-x$ and

$$
e^{-\gamma a \overline{\rho_{2}}}\left(\lambda_{n}-a-y\right)-\overline{\rho_{2}}\left(\lambda_{n}-y\right) \geq-\epsilon \bar{\zeta}\left(\lambda_{n}-y\right)
$$

for $0 \leq y \leq x$. By (3.2) and (3.3) of Lemma 3.1, we have only to prove that

$$
\sum_{j=3}^{4} \liminf _{x \rightarrow \infty} \liminf _{n \rightarrow \infty} \frac{I_{j}}{\bar{\zeta}\left(\lambda_{n}\right)} \geq 0
$$

We have

$$
\begin{aligned}
I_{3} & \geq-\epsilon \int_{0-}^{\left(\lambda_{n}-a-x\right)+} \bar{\zeta}\left(\lambda_{n}-y\right) \rho_{2}(d y) \\
& \geq-\epsilon\left(\bar{\zeta}\left(\lambda_{n}\right)+\int_{a+x}^{\lambda_{n}} \overline{\rho_{2}}\left(\lambda_{n}-y\right) \zeta(d y)\right) \\
& \geq-\epsilon\left(\bar{\zeta}\left(\lambda_{n}\right)+C_{2} \overline{\zeta^{2 *}}\left(\lambda_{n}\right)\right)
\end{aligned}
$$


and

$$
\begin{aligned}
I_{4} & \geq-\epsilon \int_{0-}^{x+} \bar{\zeta}\left(\lambda_{n}-y\right) \rho_{1}(d y) \\
& \geq-\epsilon\left(\bar{\zeta}\left(\lambda_{n}\right)+\int_{\lambda_{n}-x}^{\lambda_{n}} \overline{\rho_{1}}\left(\lambda_{n}-y\right) \zeta(d y)\right) \\
& \geq-\epsilon\left(\bar{\zeta}\left(\lambda_{n}\right)+C_{1} \overline{\zeta^{2 *}}\left(\lambda_{n}\right)\right) .
\end{aligned}
$$

Thus we see that

$$
\liminf _{n \rightarrow \infty} \frac{I_{3}}{\bar{\zeta}\left(\lambda_{n}\right)} \geq-\epsilon\left(1+C_{2} d^{*}\right),
$$

and

$$
\liminf _{n \rightarrow \infty} \frac{I_{4}}{\bar{\zeta}\left(\lambda_{n}\right)} \geq-\epsilon\left(1+C_{1} d^{*}\right) .
$$

Since $\epsilon>0$ is arbitrary, we established, for $\mathrm{j}=3,4$,

$$
\liminf _{x \rightarrow \infty} \liminf _{n \rightarrow \infty} \frac{I_{j}}{\bar{\zeta}\left(\lambda_{n}\right)} \geq 0 .
$$

Thus we have proved (3.6).

Lemma 3.4. Let $\gamma \geq 0$. Suppose that $\zeta \in \mathcal{O S} \cap \mathcal{L}(\gamma)$. For $j=1,2$, let $\rho_{j}$ be distributions on $\mathbb{R}_{+}$satisfying (3.1). Suppose further that, for every $a \geq 0$,

$$
\lim _{x \rightarrow \infty} \frac{e^{-\gamma a} \bar{\rho}_{1}(x-a)-\bar{\rho}_{1}(x)}{\bar{\zeta}(x)}=0
$$

and, for every $a \geq 0$,

$$
\lim _{x \rightarrow \infty} \frac{e^{-\gamma a} \overline{\rho_{1} * \rho_{2}}(x-a)-\overline{\rho_{1} * \rho_{2}}(x)}{\bar{\zeta}(x)}=0
$$

and that $e^{\gamma x} \rho_{1}(d x) \in \mathcal{W}$. Then, we have, for every $a \geq 0$,

$$
\lim _{x \rightarrow \infty} \frac{e^{-\gamma a} \bar{\rho}_{2}(x-a)-\bar{\rho}_{2}(x)}{\bar{\zeta}(x)}=0 .
$$

Proof. Let $\Lambda_{2}$ be the totality of increasing sequences $\left\{\lambda_{n}\right\}_{n=1}^{\infty}$ with $\lim _{n \rightarrow \infty} \lambda_{n}=$ $\infty$ such that, for every $x \in \mathbb{R}$, the following limit exists and is finite:

$$
m_{2}\left(x ;\left\{\lambda_{n}\right\}\right):=\lim _{n \rightarrow \infty} \frac{\bar{\rho}_{2}\left(\lambda_{n}-x\right)}{\bar{\zeta}\left(\lambda_{n}\right)} .
$$

We have $\Lambda_{2} \subset \Lambda$. As for $\Lambda$, it follows that, under the assumption that $\zeta \in \mathcal{O S}$ and $\overline{\rho_{2}}(x) \leq C_{2} \bar{\zeta}(x)$, there exists an increasing subsequence $\left\{\lambda_{n}\right\} \in \Lambda_{2}$ of $\left\{x_{n}\right\}$ for each sequence $\left\{x_{n}\right\}_{n=1}^{\infty}$ with $\lim _{n \rightarrow \infty} x_{n}=\infty$. Let $\left\{\lambda_{n}\right\} \in \Lambda_{2}$. Recall from Lemma 2.4 that $m\left(x ;\left\{\lambda_{n}\right\}\right)=e^{\gamma x}$ and $\widehat{\zeta}(\gamma)<\infty$. As in the proof of Lemma 3.2 , we have (3.5). We find that, for every $a \in \mathbb{R}$,

$$
\begin{aligned}
& l(x):=\lim _{n \rightarrow \infty} \frac{I_{4}}{\bar{\zeta}\left(\lambda_{n}\right)} \\
& =\int_{0-}^{x+}\left(e^{-\gamma a} m_{2}\left(a+y ;\left\{\lambda_{n}\right\}\right)-m_{2}\left(y ;\left\{\lambda_{n}\right\}\right)\right) \rho_{1}(d y) .
\end{aligned}
$$


Define $M_{2}\left(y ;\left\{\lambda_{n}\right\}\right):=e^{-\gamma y} m_{2}\left(y ;\left\{\lambda_{n}\right\}\right)$. Then $M_{2}\left(y ;\left\{\lambda_{n}\right\}\right) \leq C_{2}$ on $\mathbb{R}$. Note that

$$
l(x)=\int_{0-}^{x+}\left(M_{2}\left(a+y ;\left\{\lambda_{n}\right\}\right)-M_{2}\left(y ;\left\{\lambda_{n}\right\}\right)\right) e^{\gamma y} \rho_{1}(d y) .
$$

We see from (3.2), (3.3) of Lemma 3.1, (3.5), and (3.7) that, for every $a \in \mathbb{R}$,

$$
\lim _{x \rightarrow \infty} l(x)=\int_{0-}^{\infty}\left(M_{2}\left(a+y ;\left\{\lambda_{n}\right\}\right)-M_{2}\left(y ;\left\{\lambda_{n}\right\}\right)\right) e^{\gamma y} \rho_{1}(d y)=0 .
$$

Thus we obtain that, for every $a, b \in \mathbb{R}$,

$$
\int_{0-}^{\infty}\left(M_{2}\left(a+b+y ;\left\{\lambda_{n}\right\}\right)-M_{2}\left(b+y ;\left\{\lambda_{n}\right\}\right)\right) e^{\gamma y} \rho_{1}(d y)=0 .
$$

Since $e^{\gamma y} \rho_{1}(d y) \in \mathcal{W}$, we find from Lemma 2.7 that, for every $a \in \mathbb{R}$,

$$
M_{2}\left(a+b ;\left\{\lambda_{n}\right\}\right)=M_{2}\left(b ;\left\{\lambda_{n}\right\}\right) \text { for a.e. } b \in \mathbb{R} .
$$

Since the function $m_{2}\left(x ;\left\{\lambda_{n}\right\}\right)$ is increasing, the functions $M_{2}\left(x+;\left\{\lambda_{n}\right\}\right)$ and $M_{2}\left(x-;\left\{\lambda_{n}\right\}\right)$ exist for all $x \in \mathbb{R}$. Taking $b_{n}=b_{n}(a) \downarrow 0$ and $b_{n}=b_{n}(a) \uparrow 0$, we have

$$
M_{2}\left(a+;\left\{\lambda_{n}\right\}\right)=M_{2}\left(0+;\left\{\lambda_{n}\right\}\right) \text { and } M_{2}\left(a-;\left\{\lambda_{n}\right\}\right)=M_{2}\left(0-;\left\{\lambda_{n}\right\}\right) .
$$

As $a \uparrow 0$ in the first equality, we see that

$$
M_{2}\left(0-;\left\{\lambda_{n}\right\}\right)=M_{2}\left(0+;\left\{\lambda_{n}\right\}\right)
$$

and hence, for every $a \in \mathbb{R}$,

$$
M_{2}\left(a ;\left\{\lambda_{n}\right\}\right)=M_{2}\left(0 ;\left\{\lambda_{n}\right\}\right) .
$$

Since $\left\{\lambda_{n}\right\} \in \Lambda_{2}$ is arbitrary, we have (3.8).

Lemma 3.5. Let $\gamma \geq 0$. Suppose that $\zeta \in \mathcal{O S}$. For $j=1,2$, let $\rho_{j}$ be distributions on $\mathbb{R}_{+}$satisfying (3.1). Suppose further that, for $j=1,2$, and for every $a \geq 0$,

$$
\liminf _{x \rightarrow \infty} \frac{e^{-\gamma a} \bar{\rho}_{j}(x-a)-\bar{\rho}_{j}(x)}{\bar{\zeta}(x)} \geq 0 .
$$

If we have, for every $a \geq 0$,

$$
\lim _{x \rightarrow \infty} \frac{e^{-\gamma a} \overline{\rho_{1} * \rho_{2}}(x-a)-\overline{\rho_{1} * \rho_{2}}(x)}{\bar{\zeta}(x)}=0,
$$

then, for $j=1,2$, and for every $a \geq 0$,

$$
\lim _{x \rightarrow \infty} \frac{e^{-\gamma a} \bar{\rho}_{j}(x-a)-\bar{\rho}_{j}(x)}{\bar{\zeta}(x)}=0 .
$$

Proof. Suppose that, for some $a>0$,

$$
\limsup _{x \rightarrow \infty} \frac{e^{-\gamma a} \overline{\rho_{2}}(x-a)-\overline{\rho_{2}}(x)}{\bar{\zeta}(x)}>0 .
$$


Then there is $\left\{\lambda_{n}\right\} \in \Lambda$ such that, for some $a>0$,

$$
\lim _{n \rightarrow \infty} \frac{e^{-\gamma a} \overline{\rho_{2}}\left(\lambda_{n}-a\right)-\overline{\rho_{2}}\left(\lambda_{n}\right)}{\bar{\zeta}\left(\lambda_{n}\right)}=: \delta_{0}>0 .
$$

So there is $\delta_{1}>0$ such that, for some $a>0$,

$$
\liminf _{n \rightarrow \infty} \frac{e^{-\gamma\left(a+\delta_{1}\right)} \overline{\rho_{2}}\left(\lambda_{n}-a\right)-\overline{\rho_{2}}\left(\lambda_{n}\right)}{\bar{\zeta}\left(\lambda_{n}\right)}=: \delta_{2}>0 .
$$

Take $y_{0}$ such that $x>y_{0}>\delta_{1}$ and $\rho_{1}\left(\left(y_{0}-\delta_{1}, y_{0}\right]\right)>0$. Let $\lambda_{n}^{\prime}:=\lambda_{n}+y_{0}$ and $a^{\prime}:=a+\delta_{1}$. Then we have

$$
\begin{aligned}
& \int_{y_{0}-\delta_{1}}^{y_{0}}\left(e^{-\gamma a^{\prime}} \overline{\rho_{2}}\left(\lambda_{n}^{\prime}-a^{\prime}-y\right)-\overline{\rho_{2}}\left(\lambda_{n}^{\prime}-y\right)\right) \rho_{1}(d y) \\
& \geq \rho_{1}\left(\left(y_{0}-\delta_{1}, y_{0}\right]\right)\left(e^{-\gamma a^{\prime}} \overline{\rho_{2}}\left(\lambda_{n}-a\right)-\overline{\rho_{2}}\left(\lambda_{n}\right)\right) .
\end{aligned}
$$

Let $\lambda_{n}^{\prime}>a^{\prime}+x$ and $x>0$. Define $J$ as

$$
J:=e^{-\gamma a^{\prime}} \overline{\rho_{1} * \rho_{2}}\left(\lambda_{n}^{\prime}-a^{\prime}\right)-\overline{\rho_{1} * \rho_{2}}\left(\lambda_{n}^{\prime}\right) .
$$

Then we have as in assertion (i) of Lemma 3.1

$$
J=\sum_{j=1}^{4} I_{j}^{\prime}
$$

where

$$
\begin{gathered}
I_{1}^{\prime}:=-\int_{\lambda_{n}^{\prime}-a^{\prime}-x}^{\lambda_{n}^{\prime}-x} \overline{\rho_{1}}\left(\lambda_{n}^{\prime}-y\right) \rho_{2}(d y), \\
I_{2}^{\prime}:=\overline{\rho_{1}}(x)\left(e^{-\gamma a^{\prime}} \overline{\rho_{2}}\left(\lambda_{n}^{\prime}-a^{\prime}-x\right)-\overline{\rho_{2}}\left(\lambda_{n}^{\prime}-x\right)\right), \\
I_{3}^{\prime}:=\int_{0-}^{\left(\lambda_{n}^{\prime}-a^{\prime}-x\right)+}\left(e^{-\gamma a^{\prime}} \overline{\rho_{1}}\left(\lambda_{n}^{\prime}-a^{\prime}-y\right)-\overline{\rho_{1}}\left(\lambda_{n}^{\prime}-y\right)\right) \rho_{2}(d y),
\end{gathered}
$$

and

$$
I_{4}^{\prime}:=\int_{0-}^{x+}\left(e^{-\gamma a^{\prime}} \overline{\rho_{2}}\left(\lambda_{n}^{\prime}-a^{\prime}-y\right)-\overline{\rho_{2}}\left(\lambda_{n}^{\prime}-y\right)\right) \rho_{1}(d y) .
$$

For $1 \leq j \leq 3$, let

$$
J_{j}:=I_{j}^{\prime},
$$

and let

$$
I_{4}^{\prime}=\sum_{j=4}^{6} J_{j},
$$

where

$$
\begin{gathered}
J_{4}:=\int_{0-}^{\left(y_{0}-\delta_{1}\right)+}\left(e^{-\gamma a^{\prime}} \overline{\rho_{2}}\left(\lambda_{n}^{\prime}-a^{\prime}-y\right)-\overline{\rho_{2}}\left(\lambda_{n}^{\prime}-y\right)\right) \rho_{1}(d y), \\
J_{5}:=\int_{y_{0}}^{x}\left(e^{-\gamma a^{\prime}} \overline{\rho_{2}}\left(\lambda_{n}^{\prime}-a^{\prime}-y\right)-\overline{\rho_{2}}\left(\lambda_{n}^{\prime}-y\right)\right) \rho_{1}(d y),
\end{gathered}
$$


and

$$
J_{6}:=\int_{y_{0}-\delta_{1}}^{y_{0}}\left(e^{-\gamma a^{\prime}} \overline{\rho_{2}}\left(\lambda_{n}^{\prime}-a^{\prime}-y\right)-\overline{\rho_{2}}\left(\lambda_{n}^{\prime}-y\right)\right) \rho_{1}(d y) .
$$

Then we have

$$
J=\sum_{j=1}^{6} J_{j} .
$$

As in the proof of Lemma 3.3, we see from the assumption and (3.9) that

$$
\begin{aligned}
0 & =\lim _{n \rightarrow \infty} \frac{J}{\bar{\zeta}\left(\lambda_{n}^{\prime}\right)} \\
& \geq \sum_{j=1}^{6} \liminf _{x \rightarrow \infty} \liminf _{n \rightarrow \infty} \frac{J_{j}}{\overline{\zeta\left(\lambda_{n}^{\prime}\right)}} \\
& \geq \liminf _{n \rightarrow \infty} \frac{J_{6}}{\bar{\zeta}\left(\lambda_{n}^{\prime}\right)} \\
& \geq \liminf _{n \rightarrow \infty} \rho_{1}\left(\left(y_{0}-\delta_{1}, y_{0}\right]\right) \frac{\left(e^{-\gamma a^{\prime}} \overline{\rho_{2}}\left(\lambda_{n}-a\right)-\overline{\rho_{2}}\left(\lambda_{n}\right)\right)}{\bar{\zeta}\left(\lambda_{n}^{\prime}\right)} \\
& =\rho_{1}\left(\left(y_{0}-\delta_{1}, y_{0}\right]\right) \frac{\delta_{2}}{m\left(-y_{0} ;\left\{\lambda_{n}\right\}\right)}>0 .
\end{aligned}
$$

This is a contradiction. Thus we have, for every $a \geq 0$,

$$
\lim _{x \rightarrow \infty} \frac{e^{-\gamma a} \bar{\rho}_{2}(x-a)-\bar{\rho}_{2}(x)}{\bar{\zeta}(x)}=0 .
$$

By the analogous argument, we have for every $a \geq 0$,

$$
\lim _{x \rightarrow \infty} \frac{e^{-\gamma a} \bar{\rho}_{1}(x-a)-\bar{\rho}_{1}(x)}{\bar{\zeta}(x)}=0 .
$$

Thus we have proved the lemma.

Lemma 3.6. Let $\gamma \geq 0$. Let $\rho$ be a distribution on $\mathbb{R}_{+}$. Suppose that $\rho \in \mathcal{O S}$ and, for every $a \geq 0$,

$$
\liminf _{x \rightarrow \infty} e^{-\gamma a} \bar{\rho}(x-a) / \bar{\rho}(x) \geq 1 .
$$

Then, for some positive integer $n \geq 2, \rho^{n *} \in \mathcal{L}(\gamma)$ implies that $\rho \in \mathcal{L}(\gamma)$.

Proof. Let $\zeta:=\rho$. Then we see from Lemma 3.3 that, for every $k \in \mathbb{N}$ and every $a \geq 0$,

$$
\liminf _{x \rightarrow \infty} \frac{e^{-\gamma a} \overline{\rho^{k *}}(x-a)-\overline{\rho^{k *}}(x)}{\bar{\rho}(x)} \geq 0 .
$$

Thus we find that $\rho_{1}:=\rho$ and $\rho_{2}:=\rho^{(n-1) *}$ satisfy the assumptions of Lemma 3.5. Hence we have by Lemma 3.5, for every $a \geq 0$,

$$
\lim _{x \rightarrow \infty} \frac{e^{-\gamma a} \bar{\rho}(x-a)-\bar{\rho}(x)}{\bar{\rho}(x)}=0 .
$$

That is, $\rho \in \mathcal{L}(\gamma)$.

Remark 3.1. For $\gamma=0$, the assumption (3.10) necessarily holds, but for $\gamma>0$, without the assumption (3.10) the lemma does not hold. For $\gamma>0$, Watanabe [14] made a distribution $\eta \in \mathcal{O S}$ such that $\eta^{n *} \in \mathcal{L}(\gamma)$ for every $n \geq 2$ but $\eta \notin \mathcal{L}(\gamma)$. 


\section{Proof of results}

In this section, we prove the results stated in Sect. 1.

Lemma 4.1. Let $\gamma \geq 0$ and $\mu \in \mathcal{O S} \cap \mathcal{I D}$. If, for every $a \geq 0$, (1.1) holds, then, for all $n \in \mathbb{N}$ and every $a \geq 0$,

$$
\lim _{x \rightarrow \infty} \frac{e^{-\gamma a} \overline{\nu_{1}^{n *}}(x-a)-\overline{\nu_{1}^{n *}}(x)}{\overline{\nu_{1}^{n_{0} *}}(x)}=0,
$$

and we have $T(\mu, \gamma)=(0, \infty)$.

Proof. By induction, we see from Lemma 3.2 that if (1.1) holds for every $a \geq 0$, then, for all $n \in \mathbb{N}$ and every $a \geq 0$, we have (4.1). We have with $c:=\bar{\nu}(1)$, for $t>0$,

$$
\mu_{1}^{t *}:=e^{-c t} \sum_{k=0}^{\infty} \frac{(c t)^{k}}{k !} \nu_{1}^{k *} .
$$

Suppose that, for all $n \in \mathbb{N}$ and every $a \geq 0,(4.1)$ holds. Let $\epsilon>0$ be arbitrary. By Lemma 2.3, we can choose sufficiently large $N \in \mathbb{N}$ such that, for $\epsilon>0$,

$$
e^{-c t} \sum_{k=N+1}^{\infty} \frac{(c t)^{k}}{k !} \frac{\left|e^{-\gamma a} \overline{\nu_{1}^{k *}}(x-a)-\overline{\nu_{1}^{k *}}(x)\right|}{\overline{\nu_{1}^{n_{0} *}}(x)}<\epsilon .
$$

We find from (4.1) that, for every $a \geq 0$,

$$
\lim _{x \rightarrow \infty} e^{-c t} \sum_{k=1}^{N} \frac{(c t)^{k}}{k !} \frac{e^{-\gamma a} \overline{\nu_{1}^{k *}}(x-a)-\overline{\nu_{1}^{k *}}(x)}{\overline{\nu_{1}^{n_{0} *}}(x)}=0 .
$$

Thus we see that, for every $a \geq 0$ and for every $t>0$,

$$
\lim _{x \rightarrow \infty} \frac{e^{-\gamma a} \overline{\mu_{1}^{t *}}(x-a)-\overline{\mu_{1}^{t *}}(x)}{\overline{\nu_{1}^{n_{0 *}}}(x)}=0 .
$$

Since $\overline{\mu_{1}^{t *}}(x) \asymp \overline{\nu_{1}^{n_{0} *}}(x)$ for every $t>0$, we have $T(\mu, \gamma)=(0, \infty)$.

Lemma 4.2. Let $\gamma \geq 0$ and $\mu \in \mathcal{O S} \cap \mathcal{I D}$. If 0 is a limit point of $T(\mu, \gamma)$, then, for every $a \geq 0$, (1.1) holds.

Proof. Suppose that 0 is a limit point of $T(\mu, \gamma)$. Then, there exists a strictly decreasing sequence $\left\{t_{n}\right\}_{n=1}^{\infty}$ in $T(\mu, \gamma)$ converging to 0 as $n \rightarrow \infty$. We have with $c:=\bar{\nu}(1)$

$$
\mu_{1}^{t_{n} *}:=e^{-c t_{n}} \sum_{k=0}^{\infty} \frac{\left(c t_{n}\right)^{k}}{k !} \nu_{1}^{k *} .
$$

Since $\left\{t_{n}\right\}_{n=1}^{\infty}$ in $T(\mu, \gamma)$ and $\overline{\mu_{1}^{t_{n} *}}(x) \asymp \overline{\nu_{1}^{n_{0} *}}(x)$ from Lemma 2.2, we see that, for every $a \geq 0$,

$$
\begin{aligned}
& \lim _{x \rightarrow \infty} \frac{e^{-\gamma a} \overline{\mu_{1}^{t_{n} *}}(x-a)-\overline{\mu_{1}^{t_{n} *}}(x)}{\overline{\nu_{1}^{n_{0} *}}(x)} \\
& =\lim _{x \rightarrow \infty} \frac{e^{-\gamma a} \overline{\mu_{1}^{t_{n} *}}(x-a)-\overline{\mu_{1}^{t_{n} *}}(x)}{\overline{\mu_{1}^{t_{n} *}}(x)} \frac{\overline{\mu_{1}^{t_{n} *}}(x)}{\overline{\nu_{1}^{n_{0} *}}(x)}=0 .
\end{aligned}
$$


Thus we obtain from Lemma 2.3 that, for every $a \geq 0$,

$$
\begin{aligned}
& \limsup _{x \rightarrow \infty}\left|\frac{e^{-\gamma a} \overline{\nu_{1}}(x-a)-\overline{\nu_{1}}(x)}{\overline{\nu_{1}^{n_{0} *}}(x)}\right| \\
& =\limsup _{n \rightarrow \infty} \limsup _{x \rightarrow \infty}\left|\frac{e^{c t_{n}}}{c t_{n}} \frac{e^{-\gamma a} \overline{\mu_{1}^{t_{n} *}}(x-a)-\overline{\mu_{1}^{t_{n} *}}(x)}{\overline{\nu_{1}^{n_{0} *}}(x)}-\frac{e^{-\gamma a} \overline{\nu_{1}}(x-a)-\overline{\nu_{1}}(x)}{\overline{\nu_{1}^{n_{0} *}}(x)}\right| \\
& \leq \limsup _{n \rightarrow \infty} \limsup _{x \rightarrow \infty} \sum_{k=2}^{\infty} \frac{\left(c t_{n}\right)^{(k-1)}}{k !} \frac{e^{-\gamma a} \overline{\nu_{1}^{k *}}(x-a)+\overline{\nu_{1}^{k *}}(x)}{\overline{\nu_{1}^{n_{0} *}}(x)}=0 .
\end{aligned}
$$

Thus we have (1.1) for every $a \geq 0$.

Lemma 4.3. Let $\gamma \geq 0$ and $\mu \in \mathcal{O S} \cap \mathcal{I D}$. If $t_{0}, t_{1} \in T(\mu, \gamma)$ with $t_{1}>t_{0}$, then $t_{1}-t_{0} \in T(\mu, \gamma)$. If $T(\mu, \gamma)$ has a limit point, then $T(\mu, \gamma)=(0, \infty)$. If $T(\mu, \gamma)$ has the minimum $a_{0}>0$, then $T(\mu, \gamma)=a_{0} \mathbb{N}$.

Proof. Suppose that $t_{0}, t_{1} \in T(\mu, \gamma)$ with $t_{1}>t_{0}$. Let $\zeta:=\rho_{1}:=\mu^{t_{0} *}$ and $\rho_{2}:=\mu^{\left(t_{1}-t_{0}\right) *}$. The distribution $e^{\gamma x} \rho_{1}(d x) / \widehat{\rho}_{1}(\gamma)$ is an exponentially tilted infinitely divisible distribution and hence itself is infinitely divisible, thus having a non-vanishing characteristic function. That is, $e^{\gamma x} \rho_{1}(d x) \in \mathcal{W}$. See (iii) of Theorem 25.17 of Sato [10. Thus we see from Lemma 3.4 that $\mu^{\left(t_{1}-t_{0}\right) *} \in \mathcal{L}(\gamma)$. Thus, if $T(\mu, \gamma)$ has a limit point, then 0 is a limit point of $T(\mu, \gamma)$, and hence, by Lemmas 4.1 and $4.2, T(\mu, \gamma)=(0, \infty)$. If $T(\mu, \gamma)$ has the minimum $a_{0}>0$, then clearly $a_{0} \mathbb{N} \subset T(\mu, \gamma)$ and $T(\mu, \gamma) \backslash a_{0} \mathbb{N}=\emptyset$.

Proof of Theorem 1.1. Assertion (i) is clear from Lemmas 4.1, 4.2, and 4.3. The first part of assertion (ii) is due to Lemmas 4.1 and 4.2. Suppose that $\mu \in \mathcal{A}(\gamma)$. If $n<n_{0}$, then $\nu_{1}^{n *} \notin \mathcal{O S}$ simply because of the definition of $n_{0}$. If $n \geq n_{0}$ and $x$ is large, then $\overline{\nu_{1}^{n *}}(x) \geq \overline{\nu_{1}^{n_{0} *}}(x)$, and hence (4.1) implies that $\nu_{1}^{n *} \in \mathcal{L}(\gamma)$.

Proof of Corollary 1.1. Suppose that $\mathcal{C}(\gamma)$ is not empty. Then there is the minimum $a_{0}>0$ in $T(\mu, \gamma)$ for $\mu \in \mathcal{C}(\gamma)$. Since $a_{0}>0$ is a period of $T(\mu, \gamma)$, for $n=2, \mu^{a_{0} *}=\left(\mu^{\left(a_{0} / n\right) *}\right)^{n *} \in \mathcal{L}(\gamma)$ but $\left(\mu^{\left(a_{0} / n\right) *}\right)^{(n+1) *} \notin \mathcal{L}(\gamma)$ and $\mu^{\left(a_{0} / n\right) *} \notin \mathcal{L}(\gamma)$. Thus Hypotheses I and II are not true. Suppose that $\mathcal{C}(\gamma)$ is empty. Then, obviously, Hypotheses I and II are true. Thus (1), (2), and (3) are equivalent. We prove the equivalence of (3) and (4). Suppose that $\mathcal{C}(\gamma)$ is empty. Then for every $\mu \in \mathcal{O S} \cap \mathcal{I D}$ it holds that, for every $2 t \in T(\mu, \gamma)$, $\mu_{1}^{t *} \in \mathcal{L}(\gamma)$ and hence, for all $a \geq 0,(1.2)$ holds. Conversely, suppose that $\mathcal{C}(\gamma)$ is not empty and, for $a_{0}=2 t \in T(\mu, \gamma)$ with $\mu \in \mathcal{C}(\gamma)$ and for all $a \geq 0$, (1.2) holds. Letting $\rho_{1}:=\rho_{2}:=\mu_{1}^{t *}, \zeta:=\mu_{1}^{2 t *}$, define $\Lambda_{2}$ as in Lemma 3.4 and let $\left\{\lambda_{n}\right\} \in \Lambda_{2} \subset \Lambda$. We have (3.3) by Lemma 3.1 for $j=1,2$. We have $I_{3}+I_{4}=2 I_{4}+I_{5}$, where

$$
I_{5}:=\int_{x}^{\lambda_{n}-a-x}\left(e^{-\gamma a} \overline{\rho_{1}}\left(\lambda_{n}-a-y\right)-\overline{\rho_{1}}\left(\lambda_{n}-y\right)\right) \rho_{2}(d y),
$$

We have by the assumption (1.2) for every $a \geq 0$

$$
\limsup _{x \rightarrow \infty} \limsup _{n \rightarrow \infty} \frac{\left|I_{5}\right|}{\bar{\zeta}\left(\lambda_{n}\right)}=0
$$


Define $M_{2}\left(y ;\left\{\lambda_{n}\right\}\right):=e^{-\gamma y} m_{2}\left(y ;\left\{\lambda_{n}\right\}\right)$. Thus we find from (3.2), (3.3), and $2 t \in T(\mu, \gamma)$ that, for every $a \geq 0$,

$$
\begin{aligned}
& \lim _{x \rightarrow \infty} \lim _{n \rightarrow \infty} \frac{I_{4}}{\bar{\zeta}\left(\lambda_{n}\right)} \\
& =\int_{0-}^{\infty}\left(e^{-\gamma a} m_{2}\left(a+y ;\left\{\lambda_{n}\right\}\right)-m_{2}\left(y ;\left\{\lambda_{n}\right\}\right)\right) \rho_{1}(d y) \\
& =\int_{0-}^{\infty}\left(M_{2}\left(a+y ;\left\{\lambda_{n}\right\}\right)-M_{2}\left(y ;\left\{\lambda_{n}\right\}\right)\right) e^{\gamma y} \rho_{1}(d y)=0 .
\end{aligned}
$$

The distribution $e^{\gamma x} \rho_{1}(d x) / \widehat{\rho}_{1}(\gamma)$ is an exponentially tilted infinitely divisible distribution and hence itself is infinitely divisible, thus having a non-vanishing characteristic function. That is,

$$
e^{\gamma y} \rho_{1}(d y)=e^{\gamma y} \mu_{1}^{t *}(d y) \in \mathcal{W} .
$$

As in the proof of Lemma 3.4, we have $\rho_{2}=\mu_{1}^{t *} \in \mathcal{L}(\gamma)$. This is a contradiction. Thus (3) and (4) are equivalent.

Proof of Remark 1.1. Let $\gamma=0$. Then we see from Lemma 3.6 that Hypothesis II is true. Thus $\mathcal{C}(0)$ is empty and hence Remark 1.1 follows from Theorem 1.1 .

Proof of Proposition 1.1. Let $\gamma>0$ and $\mu \in \mathcal{O S} \cap \mathcal{I D}$. Suppose that (1.3) holds for every $a \geq 0$. Let $\zeta:=\nu_{1}^{n_{0} *}$. Then, by induction, we see from (1.3) and Lemma 3.3 that, for every $n \in \mathbb{N}$ and every $a \geq 0$,

$$
\liminf _{x \rightarrow \infty} \frac{e^{-\gamma a} \overline{\nu_{1}^{n *}}(x-a)-\overline{\nu_{1}^{n *}}(x)}{\overline{\nu_{1}^{n_{0} *}}(x)} \geq 0 .
$$

Let $\epsilon>0$ be arbitrary. Thus, letting $N \in \mathbb{N}$ sufficiently large, we have, for every $t>0$ and for every $a \geq 0$,

$$
\begin{aligned}
& \liminf _{x \rightarrow \infty} \frac{e^{-\gamma a} \overline{\mu_{1}^{t *}}(x-a)-\overline{\mu_{1}^{t *}}(x)}{\overline{\nu_{1}^{n_{0} *}}(x)} \\
& =\liminf _{x \rightarrow \infty} e^{-c t} \sum_{k=1}^{N} \frac{(c t)^{k}}{k !} \frac{e^{-\gamma a} \overline{\nu_{1}^{k *}}(x-a)-\overline{\nu_{1}^{k *}}(x)}{\overline{\nu_{1}^{n_{0} *}}(x)} \\
& -\limsup _{x \rightarrow \infty} e^{-c t} \sum_{k=N+1}^{\infty} \frac{(c t)^{k}}{k !} \frac{e^{-\gamma a} \overline{\nu_{1}^{k *}}(x-a)+\overline{\nu_{1}^{k *}}(x)}{\overline{\nu_{1}^{n_{0} *}}(x)} \geq-\epsilon .
\end{aligned}
$$

Since $\epsilon>0$ is arbitrary and $\overline{\nu_{1}^{n_{0} *}}(x) \asymp \overline{\mu_{1}^{(t / n) *}}(x)$ for every $n \in \mathbb{N}$, we obtain that $\rho:=\mu_{1}^{(t / n) *}$ satisfies $\rho \in \mathcal{O S}$ and (3.10) holds. Hence we find from Lemma 3.6 that if $t \in T(\mu, \gamma)$, then $t / n \in T(\mu, \gamma)$ for every $n \in \mathbb{N}$. Thus, by Lemmas 4.1 and 4.2 , either $T(\mu, \gamma)=(0, \infty)$ or $\emptyset$.

Proof of Proposition 1.2. Suppose that $\nu_{1}^{2 *} \in \mathcal{L}(\gamma)$ and the real part of $\widehat{\nu}_{1}(\gamma+i z)$ is not 0 for every $z \in \mathbb{R}$. If $t \in T(\mu, \gamma)$, then

$$
\mu_{1}^{t *}=e^{-c t} \sum_{k=0}^{\infty} \frac{(c t)^{k}}{k !} \nu_{1}^{k *} \in \mathcal{L}(\gamma) \cap \mathcal{O S} .
$$


Define distributions $\eta_{1}$ and $\eta_{2}$ on $\mathbb{R}_{+}$as

$$
\eta_{1}:=(\cosh (c t))^{-1} \sum_{k=0}^{\infty} \frac{(c t)^{2 k}}{(2 k) !} \nu_{1}^{(2 k) *}
$$

and

$$
\eta_{2}:=(\sinh (c t))^{-1} \sum_{k=0}^{\infty} \frac{(c t)^{2 k+1}}{(2 k+1) !} \nu_{1}^{(2 k+1) *} .
$$

We see from Proposition 3.1 of Shimura and Watanabe [11] that $\eta_{j} \in \mathcal{O S}$ and $\overline{\eta_{j}}(x) \asymp \overline{\nu_{1}^{n_{0} *}}(x)$ for $j=1,2$. Let $\epsilon>0$ be arbitrary. We obtain from Lemma 2.3 that there is a positive integer $N=N(a, \epsilon, t)$ such that

$$
\limsup _{x \rightarrow \infty}(\cosh (c t))^{-1} \sum_{k=N+1}^{\infty} \frac{(c t)^{2 k}}{(2 k) !} \frac{e^{-\gamma a} \overline{\nu_{1}^{(2 k) *}}(x-a)+\overline{\nu_{1}^{(2 k) *}}(x)}{\overline{\nu_{1}^{n_{0 *}}}(x)}<\epsilon .
$$

Since $\nu_{1}^{(2 k) *} \in \mathcal{L}(\gamma)$ for every $k \geq 0$, we have, for every $a \geq 0$ and every $t>0$,

$$
\limsup _{x \rightarrow \infty}(\cosh (c t))^{-1} \sum_{k=0}^{N} \frac{(c t)^{2 k}}{(2 k) !} \frac{\left|e^{-\gamma a} \overline{\nu_{1}^{(2 k) *}}(x-a)-\overline{\nu_{1}^{(2 k) *}}(x)\right|}{\overline{\nu_{1}^{n_{0} *}}(x)}=0 .
$$

Thus with some $C=C(t)>0$ we have, for every $a \geq 0$ and every $t>0$,

$$
\begin{aligned}
& \limsup _{x \rightarrow \infty} \frac{\left|e^{-\gamma a} \overline{\eta_{1}}(x-a)-\overline{\eta_{1}}(x)\right|}{\overline{\eta_{1}}(x)} \\
& \leq \limsup _{x \rightarrow \infty}(\cosh (c t))^{-1} \sum_{k=0}^{N} \frac{(c t)^{2 k}}{(2 k) !} \frac{\left|e^{-\gamma a} \overline{\nu_{1}^{(2 k) *}}(x-a)-\overline{\nu_{1}^{(2 k) *}}(x)\right|}{C \overline{\nu_{1}^{n_{0} *}}(x)} \\
& +\limsup _{x \rightarrow \infty}(\cosh (c t))^{-1} \sum_{k=N+1}^{\infty} \frac{(c t)^{2 k}}{(2 k) !} \frac{e^{-\gamma a} \overline{\nu_{1}^{(2 k) *}}(x-a)+\overline{\nu_{1}^{(2 k) *}}(x)}{C \overline{\nu_{1}^{n_{0} *}}(x)} \\
& \leq \epsilon / C .
\end{aligned}
$$

Since $\epsilon>0$ is arbitrary, we have

$$
\eta_{1} \in \mathcal{L}(\gamma) \cap \mathcal{O S}
$$

Since

$$
\sinh (c t) \eta_{2}=e^{c t} \mu_{1}^{t *}-\cosh (c t) \eta_{1},
$$

we have by (4.2) and (4.3)

$$
\eta_{2} \in \mathcal{L}(\gamma) \cap \mathcal{O S}
$$

Let $\zeta:=\rho_{1}:=\eta_{2}$ and $\rho_{2}:=\nu_{1}$. Then, by argument similar to the proof of (4.3),

$$
\rho_{1} * \rho_{2}=(\sinh (c t))^{-1} \sum_{k=0}^{\infty} \frac{(c t)^{2 k+1}}{(2 k+1) !} \nu_{1}^{(2 k+2) *} \in \mathcal{L}(\gamma) \cap \mathcal{O S} .
$$

Since the real part of $\widehat{\nu}_{1}(\gamma+i z)$ is not 0 for every $z \in \mathbb{R}$,

$$
2 \sinh (c t) \widehat{\rho}_{1}(\gamma+i z)=\exp \left(c t \widehat{\nu}_{1}(\gamma+i z)\right)-\exp \left(-c t \widehat{\nu}_{1}(\gamma+i z)\right) \neq 0
$$


for every $z \in \mathbb{R}$, that is, $e^{\gamma x} \rho_{1}(d x) \in \mathcal{W}$. Thus we see from Lemma 3.4 that

$$
\lim _{x \rightarrow \infty} \frac{e^{-\gamma a} \bar{\nu}_{1}(x-a)-\bar{\nu}_{1}(x)}{\bar{\zeta}(x)}=0 .
$$

Since $\bar{\zeta}(x) \asymp \overline{\nu_{1}^{n_{0 *}}}(x)$, we see from Theorem 1.1 that $T(\mu, \gamma)=(0, \infty)$. Thus we have proved the proposition.

Proof of Proposition 1.3. Let $\gamma>0$ and $\mu \in \mathcal{O S} \cap \mathcal{I D}$. Suppose that $\nu_{1}^{n_{1} *} \in \mathcal{S}_{\sharp}$. Since $\overline{\mu^{t *}}(x) \asymp \overline{\nu_{1}^{n_{1} *}}(x)$, we have $\mu^{t *} \in \mathcal{S}_{\sharp}$ for every $t>0$. Thus we see from Lemmas 2.5 and 2.6 that if $T(\mu, \gamma) \neq \emptyset$, then $\nu_{1} \in \mathcal{S}(\gamma)$ and hence $T(\mu, \gamma)=(0, \infty)$. That is, either $T(\mu, \gamma)=(0, \infty)$ or $\emptyset$. Moreover, $T(\mu, \gamma)=(0, \infty)$ if and only if $\nu_{1} \in \mathcal{S}(\gamma)$.

\section{Acknowledgements}

The author is grateful to the referee for his careful reading the manuscript and helpful advice.

\section{References}

[1] Cui, Z., Wang, Y., Xu, H. : Some positive conclusions related to the Embrechts-Goldie conjecture. arXiv:1609.00912 (2016).

[2] Embrechts, P., Goldie, C. M. : On closure and factorization properties of subexponential and related distributions. J. Aust. Math. Soc. A 29 (1980) 243256 .

[3] Embrechts, P., Goldie, C. M. : On convolution tails. Stochastic Process. Appl. 13 (1982) 263278.

[4] Embrechts, P., Goldie, C.M., Veraverbeke, N.: Subexponentiality and infinite divisibility. Z. Wahrscheinlichkeitstheorie Verw. Gebiet. 49 (1979) 335347.

[5] Klüppelberg, C. : Asymptotic ordering of distribution functions on convolution semigroup. Semigroup Forum. 40 (1990) 7792.

[6] Klüppelberg, C., Villasenor, J. A. : The full solution of the convolution closure problem for convolution-equivalent distributions. J. Math. Anal. Appl. 160 (1991) 79-92.

[7] Leslie, J. R. : On the non-closure under convolution of the subexponential family. J. Appl. Probab. 26 (1989) 58-66.

[8] Pakes, A.G.: Convolution equivalence and infinite divisibility. J. Appl. Probab. 41 (2004) 407-424.

[9] Pakes, A. G. : Convolution equivalence and infinite divisibility: Corrections and corollaries. J. Appl. Probab. 44 (2007) 295305.

[10] Sato, K.: Lévy processes and infinitely divisible distributions. Cambridge Studies in Advanced Mathematics, 68 Cambridge Univ. Press. 2013. 
[11] Shimura, T., Watanabe, T.: Infinite divisibility and generalized subexponentiality. Bernoulli 11 (2005) 445-469.

[12] Shimura, T., Watanabe, T. : On the convolution roots in the convolutionequivalent class. The Institute of Statistical Mathematics Cooperative Research Report 175 (2005) pp. 115.

[13] Watanabe, T.: Convolution equivalence and distributions of random sums. Probab. Theory Related Fields 142 (2008) 367-397.

[14] Watanabe, T.: The Wiener condition and the conjectures of Embrechts and Goldie. Ann. Probab. 47 (2019) 12211239.

[15] Watanabe, T., Yamamuro, K.: Ratio of the tail of an infinitely divisible distribution on the line to that of its Lévy measure. Electron. J. Probab. 15 (2010) 44-74.

[16] Watanabe, T., Yamamuro, K.: Two non-closure properties on the class of subexponential densities. J. Theoret. Probab. 30 (2017) 1059-1075.

[17] Wiener, N.: Tauberian theorems. Ann. of Math. (2)33 (1932) 1100.

[18] Xu, H., Foss, S., Wang, Y. : Convolution and convolution-root properties of longtailed distributions. Extremes 18 (2015) 605628.

[19] Xu, H., Wang, Y., Cheng, D., Yu, C.: On the closure under convolution roots related to an infinitely divisible distribution in the distribution class $\mathcal{L}(\gamma)$. arXiv:1512.01792 (2015). 